\title{
Progressive Aerodynamic Model Identification From Dynamic Water Tunnel Test of the F-16XL Aircraft
}

\author{
Patrick C. Murphy* \\ NASA Langley Research Center, Hampton, VA, 23681-2199 \\ Vladislav Klein ${ }^{\dagger}$ and Nathan M. Szyba ${ }^{\ddagger}$ \\ The George Washington University, NASA Langley Research Center, Hampton, VA, 23681-2199
}

\begin{abstract}
Development of a general aerodynamic model that is adequate for predicting the forces and moments in the nonlinear and unsteady portions of the flight envelope has not been accomplished to a satisfactory degree. Predicting aerodynamic response during arbitrary motion of an aircraft over the complete flight envelope requires further development of the mathematical model and the associated methods for ground-based testing in order to allow identification of the model. In this study, a general nonlinear unsteady aerodynamic model is presented, followed by a summary of a linear modeling methodology that includes test and identification methods, and then a progressive series of steps suggesting a roadmap to develop a general nonlinear methodology that defines modeling, testing, and identification methods. Initial steps of the general methodology were applied to static and oscillatory test data to identify rolling-moment coefficient. Static measurements uncovered complicated dependencies of the aerodynamic coefficient on angle of attack and sideslip in the stall region making it difficult to find a simple analytical expression for the measurement data. In order to assess the effect of sideslip on the damping and unsteady terms, oscillatory tests in roll were conducted at different values of an initial offset in sideslip. Candidate runs for analyses were selected where higher order harmonics were required for the model and where inphase and out-of-phase components varied with frequency. From these results it was found that only data in the angle-of-attack range of $35^{\circ}$ to $37.5^{\circ}$ met these requirements. From the limited results it was observed that the identified models fit the data well and both the damping-in-roll and the unsteady term gain are decreasing with increasing sideslip and motion amplitude. Limited similarity between parameter values in the nonlinear model and the linear model suggest that identifiability of parameters in both terms may be a problem. However, the proposed methodology can still be used with careful experiment design and carefully selected values of angle of attack, sideslip, amplitude, and frequency of the oscillatory data.
\end{abstract}

\section{Nomenclature}

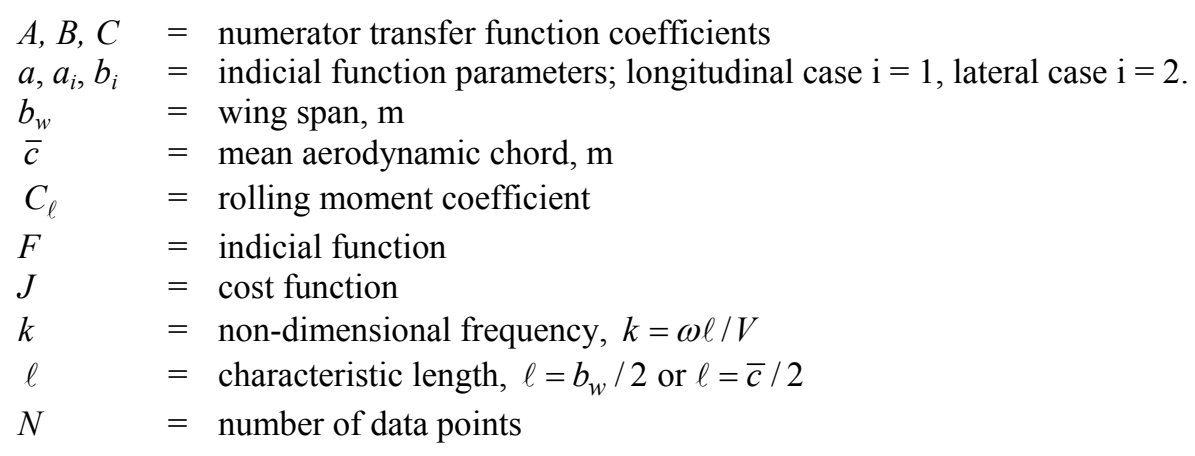

\footnotetext{
${ }^{*}$ Senior Research Engineer, Airborne Systems, Mail Stop 132, Associate Fellow.

${ }^{\dagger}$ Professor Emeritus, Airborne Systems, Mail Stop 132, Associate Fellow.

* Graduate Research Assistant, Airborne Systems, Mail Stop 132, student member.
} 


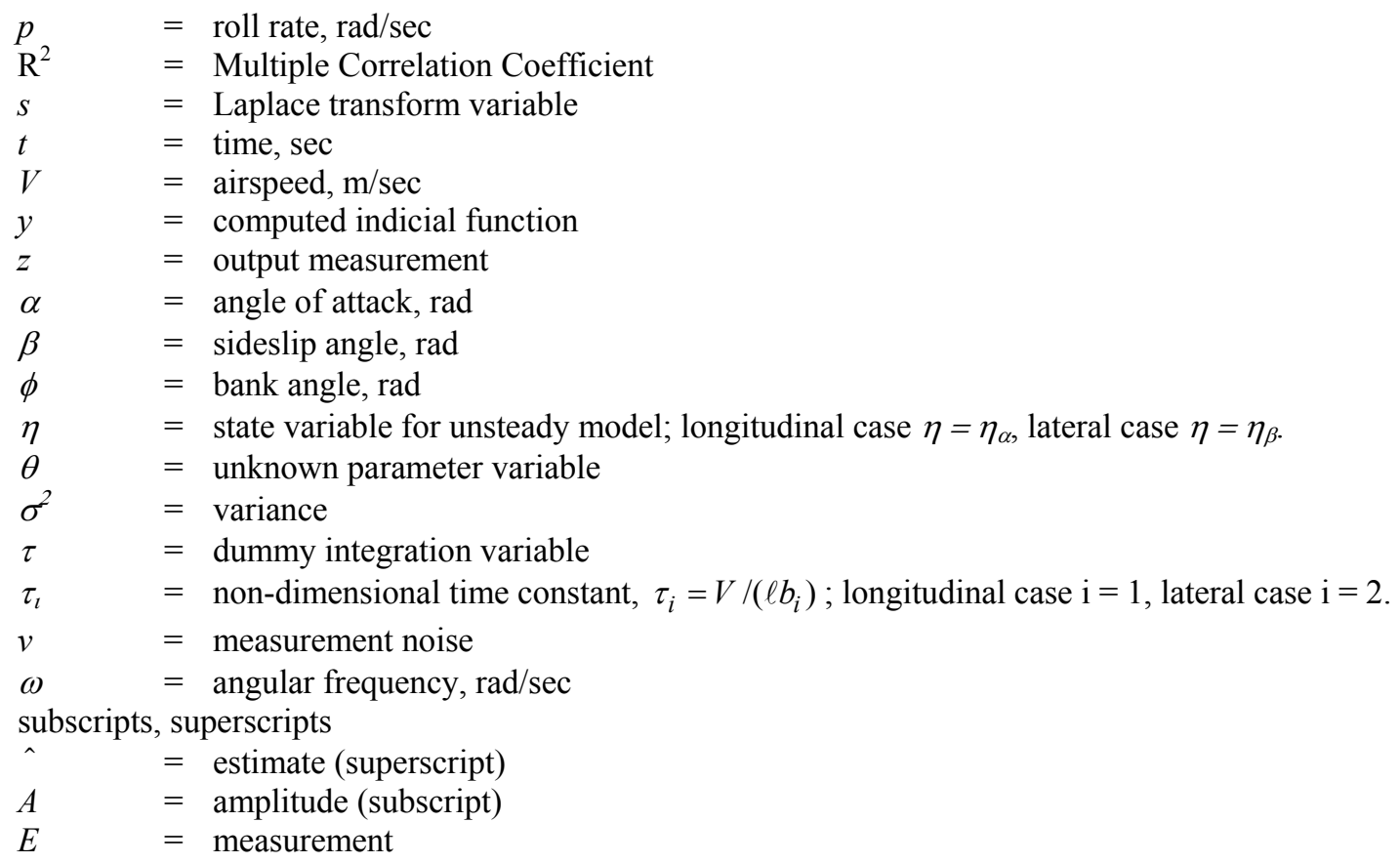

Aerodynamic Derivatives

$C_{a \xi_{1}}(\infty) \equiv C_{a \xi_{1}}=\frac{\partial C_{a}}{\partial \xi_{1}}$ where $a=\left(C_{D}, C_{L}, C_{Y}\right)$ or $\left(C_{m}, C_{\ell}, C_{n}\right)$ and $\xi_{1}=(\alpha, \beta)$
$C_{a \xi_{2}}(\infty) \equiv C_{a \xi_{2}}=\frac{\partial C_{a}}{\partial \xi_{2}}$ where $a=\left(C_{D}, C_{L}, C_{Y}\right)$ or $\left(C_{m}, C_{\ell}, C_{n}\right)$ and $\xi_{2}=(p, q, \mathrm{r})$

\section{Introduction}

$\mathrm{D}$ EVELOPMENT of a general aerodynamic model that is adequate for predicting forces and moments in nonlinear and unsteady portions of the flight envelope has not been accomplished to a satisfactory degree. Aerodynamic phenomena such as shock waves, separated flows, and vortex flows can cause faulty prediction of aircraft response when conventional linear aerodynamic models are used. Predicting aerodynamic response during arbitrary motion of an aircraft over the complete flight envelope requires further development of the mathematical model and associated methods for testing that will allow identification of the model.

Development of the aircraft aerodynamic model began in the early days of flight. Byran's formulation ${ }^{1}$ in 1911 established the conventional assumption that aerodynamic forces and moments depend only on instantaneous values of aircraft states and can be expressed in a linear polynomial expansion. These assumptions work well, depending on the particular configuration, but are primarily restricted to linear portions of the flight envelope. Adding nonlinear terms to the series expansion ${ }^{2}$ extends the capability of the model allowing a certain class of model nonlinearity to enter the response but still no memory of previous states is captured. Improving this formulation to accommodate unsteady responses requires additional differential equations to capture the lag in response. Research efforts addressing the nonlinear unsteady problem began in the 1970's with recognition that the Volterra functional was a desirable mathematical structure ${ }^{3}$. This model structure, in the form of indicial models, is sufficiently general to capture a large class of nonlinear unsteady behaviors. A number of researchers have investigated this modeling problem. The modeling of unsteady aerodynamic characteristics of an aircraft from experimental data was first reported in Ref. 4. The model equations were formulated as state-space equations. Further improvement to this modeling and parameter estimation followed in Refs. 5-7. Many other researchers have approached the modeling problem using an indicial function formulation. In Refs. 8-10, linear models were applied to wind tunnel data from different testing facilities for different aircraft models. In Refs. 11 and 12, nonlinear indicial response models were applied to the rolling 65-degree delta wing and in Ref. 13 to the prediction of a dynamically stalling wing. Nonlinear indicial models were also used in model identification from wind tunnel oscillatory data using least squares and maximum likelihood principles ${ }^{14}$. In Ref. 15 a methodology was introduced that allowed efficient identification of a linear unsteady model for an F-16XL configuration in planar forced oscillation. In this approach the aerodynamic 
model was formulated with static and steady-flow damping terms separate from unsteady indicial components. Enhanced identification and efficient testing was accomplished by using wide-band inputs. In Ref. 16 additional experiments were performed to validate the methodology, mathematical model, and test techniques used in Ref. 15.

The present study extends the work in Ref. 16 as part of the ongoing effort at NASA Langley Research Center (LaRC) to develop a more general aerodynamic model and associated test methods. To date, development and application of the indicial model methodology at LaRC has been done with a number of limiting assumptions. This is a natural result of the research progressing from specific simpler models to more general complex models. In this paper, a general nonlinear unsteady aerodynamic model is presented, followed by a summary of a linear modeling methodology that includes test and identification methods. Then a progressive series of steps that remove limiting assumptions and suggest a roadmap toward development of a general nonlinear methodology are presented. Included in this paper are results from application of two initial steps of the general methodology applied to static and oscillatory test data to identify rolling-moment coefficient.

The general methodology for testing and data analysis is being developed using data from both wind tunnel and water tunnel tests. Results presented in this paper are taken from water tunnel experiments. This choice provides data at relatively low cost and does not affect conclusions drawn regarding the methodology. Tests are done, under a Phase III SBIR with NASA LaRC, using an advanced dynamic test rig at Rolling Hills Research Company.

\section{General Aerodynamic Model}

Assumptions used for this general model impose only a few limitations. For this case only rigid-body responses are considered and relatively constant flight path properties (e.g., density, viscosity, dynamic pressure) are assumed. And assuming that the aerodynamic coefficients are single-valued functions of aircraft state variables $\alpha, \beta, \mathrm{p}, \mathrm{q}$, and $\mathrm{r}$, then according to Ref. 3, these coefficients can be formulated as

$$
\begin{array}{r}
C_{a}(t)=C_{a}(0)+\int_{0}^{t} C_{a_{\xi_{1}}}(t-\tau ; \xi(\tau))^{T} \frac{d}{d \tau} \xi_{1}(\tau) d \tau \\
+\frac{l}{V} \int_{0}^{t} C_{a \xi_{2}}(t-\tau ; \xi(\tau))^{T} \frac{d}{d \tau} \xi_{2}(\tau) d \tau
\end{array}
$$

where $C_{a}(t)$ is a coefficient of aerodynamic force or moment, $C_{a}(0)$ is the value of the coefficient at initial steadystate conditions, and $C_{a \xi}(t)$ is a vector of indicial functions whose elements are the responses in $C_{a}$ to unit steps in $\xi$. $\xi$ is defined as

$$
\xi=\left[\xi_{1} \vdots \xi_{2}\right]=\left[\begin{array}{llll}
\alpha & \beta \vdots p & q & r
\end{array}\right]^{T}
$$

and $\ell$ is the characteristic length $\left(\ell=\bar{c} / 2\right.$ or $\left.\ell=b_{w} / 2\right)$. The indicial functions approach steady-state values with increasing values of the argument $(t-\tau)$. To indicate this property, each indicial function can be expressed as

$$
C_{a_{\xi j}}(t-\tau ; \xi(\tau))=C_{a_{\xi j}}(\infty ; \xi(\tau))-F_{a_{\xi j}}(t-\tau ; \xi(\tau))
$$

where $C_{a_{j}}(\infty ; \xi(\tau))$ is the rate of change of the coefficient $C_{a}$ with $\xi_{j}$, while the remaining variables $\xi$ are fixed at the instantaneous values of $\xi(\tau)$, and $F_{a_{\xi}}$ is the deficiency function. This function approaches zero for $(t-\tau) \rightarrow \infty$. When Eq. (2) is substituted into Eq. (1) the terms involving the steady-state parameters can be integrated and Eq. (1) becomes 


$$
\begin{array}{r}
C_{a}(t)=C_{a}(\infty ; \xi(t))-\int_{0}^{t} F_{a_{\xi_{1}}}(t-\tau ; \xi(\tau))^{T} \frac{d}{d \tau} \xi_{1}(\tau) d \tau \\
-\frac{l}{V} \int_{0}^{t} F_{a_{\xi_{2}}}(t-\tau ; \xi(\tau))^{T} \frac{d}{d \tau} \xi_{2}(\tau) d \tau
\end{array}
$$

where $C_{a}(\infty ; \xi(t))$ is the total aerodynamic coefficient that would correspond to steady flow with $\xi$ fixed at the instantaneous values $\xi(t) . F_{a_{\xi_{1}}}$ and $F_{a_{\xi_{2}}}$ are vectors of deficiency functions. Further simplification of Eq. (3) is achieved by assuming that the coefficients $C_{a}$ are linearly dependent on the angular rates $\xi_{2}$. Applying a Taylor series expansion about $\xi_{2}=0$ to terms in Eq. (3) and keeping only the linear terms results in

$$
\begin{array}{r}
C_{a}(t)=C_{a}\left(\infty ; \xi_{1}(t), 0\right)+\frac{l}{V} \frac{\partial C_{a}}{\partial \xi_{2}}\left(\infty ; \xi_{1}(t), 0\right)^{T} \xi_{2} \\
-\int_{0}^{t} F_{a \xi_{1}}\left(t-\tau ; \xi_{1}(\tau), 0\right)^{T} \frac{d}{d \tau} \xi_{1}(\tau) d \tau
\end{array}
$$

or in simplified notation

$$
\begin{array}{r}
C_{a}(t)=C_{a}(\infty ; \alpha(t), \beta(t))+\frac{l}{V} C_{a_{p}}(\infty ; \alpha, \beta) p(t) \\
+\frac{l}{V} C_{a_{q}}(\infty ; \alpha, \beta) q+\frac{l}{V} C_{a_{r}}(\infty ; \alpha, \beta) r(t) \\
-\int_{0}^{t} F_{a_{\alpha}}(t-\tau ; \alpha(\tau), \beta(\tau)) \dot{\alpha}(\tau) d \tau \\
-\int_{0}^{t} F_{a_{\beta}}(t-\tau ; \alpha(\tau), \beta(\tau)) \dot{\beta}(\tau) d \tau
\end{array}
$$

where

$$
C_{a_{p}}=\frac{\partial C_{a}}{\partial \frac{p b}{2 V}}, C_{a_{q}}=\frac{\partial C_{a}}{\partial \frac{q \bar{c}}{2 V}} \text {, and } C_{a_{r}}=\frac{\partial C_{a}}{\partial \frac{r b}{2 V}}
$$

The deficiency function in Eq. (4) is postulated as

$$
\begin{gathered}
F(t ; \alpha, \beta)=h(t ; \alpha, \beta) a(\alpha, \beta) \\
h(t ; \alpha, \beta)=e^{-b(\alpha, \beta) t}
\end{gathered}
$$

where $a(\alpha, \beta)$ and $b(\alpha, \beta)$ are polynomials in $(\alpha, \beta)$. When the differential operator, $D \equiv d / d t$, is introduced, then the first convolution integral in Eq. (4) can be expressed as 


$$
\int_{0}^{t} F_{a_{\alpha}}(t-\tau ; \alpha(\tau), \beta(\tau)) \dot{\alpha}(\tau) d \tau=\frac{a_{1}(\alpha, \beta)}{D+b_{1}(\alpha, \beta)} D \alpha(t)=\eta_{\alpha}(t)
$$

and similarly for the second convolution integral

$$
\frac{a_{2}(\alpha, \beta)}{D+b_{2}(\alpha, \beta)} D \beta(t)=\eta_{\beta}(t)
$$

Equations (8) and (9) represent first-order linear differential equations

$$
\dot{\eta}_{\alpha}(t)+b_{1}(\alpha, \beta) \eta_{\alpha}(t)=a_{1}(\alpha, \beta) \dot{\alpha}
$$

and similarly for $\eta_{\beta}(t)$. Substituting Eq. (8) and Eq. (9) into Eq. (4) results in

$$
\begin{gathered}
C_{a}(t)=C_{a}(\infty ; \alpha, \beta)+\frac{l}{V} C_{a_{p}}(\infty ; \alpha, \beta) p(t) \\
+\frac{l}{V} C_{a_{q}}(\infty ; \alpha, \beta) q(t)+\frac{l}{V} C_{a_{r}}(\infty ; \alpha, \beta) r(t) \\
-\frac{a_{1}(\alpha, \beta)}{D+b_{1}(\alpha, \beta)} D \alpha(t)-\frac{a_{2}(\alpha, \beta)}{D+b_{2}(\alpha, \beta)} D \beta(t)
\end{gathered}
$$

\section{Linear Model Identification Methodology}

Linear model equations can be obtained from Eq. (11) by considering small deviations in state variables from steady-state trim conditions. Then $C_{L}, C_{D}$, and $C_{m}$ can be expressed as linear functions of $(\alpha, q)$ and $C_{Y}, C_{\ell}$, and $C_{n}$ as linear functions of $(\beta, p, r)$. The resulting equation for the longitudinal aerodynamic coefficient is

$$
\begin{array}{r}
C_{a}=C_{a}(0)+C_{a_{\alpha}} \alpha+\frac{l}{V} C_{a_{q}} q \\
-\int_{0}^{t} F_{a_{\alpha}}(t-\tau) \dot{\alpha}(\tau) d \tau
\end{array}
$$

and for the lateral coefficient

$$
\begin{array}{r}
C_{a}=C_{a}(0)+C_{a_{\beta}} \beta+\frac{l}{V} C_{a_{p}} p+\frac{l}{V} C_{a_{r}} r \\
-\int_{0}^{t} F_{a_{\beta}}(t-\tau) \dot{\beta}(\tau) d \tau
\end{array}
$$

where 


$$
\begin{aligned}
& F_{a_{\alpha}}=a_{1} e^{-b_{1} t} \\
& F_{a_{\beta}}=a_{2} e^{-b_{2} t} .
\end{aligned}
$$

Rewriting Eq. (12a) with the state space form suggested by Eqs. (8) and (10) the linear state space form is

$$
\begin{aligned}
& \dot{\eta}(t)=-b_{1} \eta+a \dot{\alpha} \\
& C_{a}(t)=C_{a_{\alpha}}(\infty) \alpha(t)+\frac{l}{V} C_{a_{q}}(\infty) q(t)-\eta(t) .
\end{aligned}
$$

The four unknown parameters, $C_{a_{\alpha}}(\infty), C_{a_{q}}(\infty), a, b_{1}$, in Eq. (13) can be estimated, in principle, from measurement time histories of $C_{a}, \alpha, \dot{\alpha}$ and $q$. If these data are obtained from a one-degree-of-freedom oscillatory test in the wind tunnel (or water tunnel), then $\dot{\alpha}=q$ and the aerodynamic model equations can be expressed in the form of a transfer function

$$
\frac{C_{a}(s)}{\alpha(s)}=\frac{A s^{2}+B s+C}{s+b_{1}}
$$

where $s$ is the Laplace transform parameter and

$$
\begin{aligned}
A & =\frac{\ell}{V} C_{a_{q}}(\infty) \\
B & =C_{a_{\alpha}}(\infty)-a+b_{1} \frac{\ell}{V} C_{a_{q}}(\infty) . \\
C & =b_{1} C_{a_{\alpha}}(\infty)
\end{aligned}
$$

There have been several estimation techniques suggested for parameter estimation from measured data. In Ref. 16 three different methods were used, 2-Step regression in the time domain, regression and maximum likelihood in the frequency domain. In the 2-Step regression parameter $b_{1}$ is estimated first and then used as a known parameter in estimation of the three remaining parameters. The cost functions for the linear regression and maximum likelihood methods, respectively, have the forms

$$
J(\theta)=\sum_{i=1}^{N}\left|C_{a}(i)\left(b_{1}+j \omega_{i}\right)+\left(A \omega_{i}^{2}-C-j B \omega_{i}\right) \alpha(i)\right|^{2}
$$

and

$$
J(\theta)=\sum_{i=1}^{N}\left|C_{a}(i)-\frac{A \omega_{i}^{2}-C-j B \omega_{i}}{b_{1}+j \omega_{i}} \alpha(i)\right|^{2}
$$

where $N$ is the number of data points. Parameters are then obtained from Eq. (15).

Oscillatory tests using a simple harmonic input are usually repeated at different frequencies. If the data are to be used to estimate (four) unsteady model parameters then six or more frequencies are recommended for better 
statistical results. In order to avoid the large number of runs, the use of a wide-band input is recommended ${ }^{15}$. Specifically, the Schroeder sweep ${ }^{17}$ was selected with specified amplitude to provide a flat power spectrum over a specified frequency range. Transforms of the time histories of the input and aerodynamic coefficients to the frequency domain were accomplished using a Discrete Fourier Transform (DFT) algorithm ${ }^{18}$. This algorithm utilizes a zoom transform and allows the transform to be performed over the frequency range corresponding to the wideband input.

\section{Nonlinear Model Identification Methodology}

A nonlinear modeling methodology that defines the mathematical model, algorithms for identification, and required test techniques is presented in this section only as a first candidate toward developing a more complete and general nonlinear methodology. Three steps in the candidate methodology suggest a progression for developing a general methodology. The modeling philosophy behind the progression is to utilize the least complex model that provides an adequate representation of the aerodynamic response; basically an application of the principle of parsimony. With this philosophy, a progression is made toward more complex models and correspondingly more complex experiment design. The simplifying assumptions associated with the least complex model are removed in progression, as required to achieve model adequacy. Before progressing through a series of dynamic tests, a complete static model is required to identify regions where static nonlinearities exist and to define the static term for the model.

Step 1. In the first series of dynamic tests, a linear model methodology is applied. Dynamic tests begin with single-frequency, one-degree-of-freedom, small-amplitude motions including constant nonzero sideslip during longitudinal motions and nonzero mean sideslip during lateral-directional motions over a large range of angle of attack and sideslip. Inherent in these tests is the simplifying assumption of superposition, so longitudinal and lateral axes are treated independently. Using harmonic analysis ${ }^{19}$ complexity of the aerodynamic response is assessed to determine where the least complex model can be applied with sufficient adequacy or equivalently to determine which simplifying assumptions are violated, if any. In general, it is expected that with small-amplitude maneuvers only a conventional linear response model or a linear unsteady model will be required and therefore only a $1^{\text {st }}$ order harmonic model (conventional in-phase and out-of-phase coefficients) will result from harmonic analysis. If a linear model is adequate then the linear model methodology applies and can be used to identify the full linear unsteady model from either single-frequency ${ }^{10}$ or wide-band forced-oscillation tests ${ }^{16}$. If the linear model is not adequate then a more general model structure must be utilized. One indicator of model adequacy is the Multiple Correlation Coefficient $\left(R^{2}\right)$. The metric value varies between 0 and 1 and is reduced from 1 as the output signal fails to be represented by the model. This metric can be computed as part of the parameter estimation process and can be expressed as

$$
R^{2}=1-\frac{\sum_{i}[z(i)-\hat{y}(i)]^{2}}{\sum_{i}[z(i)-\bar{z}]^{2}}, 0<R^{2}<1
$$

where

$$
\bar{z}=\frac{1}{N} \sum_{i} z(i)
$$

and $\hat{y}(i)$ is the computed value of the measurement $z(i)$. An important motivation for this step is that it reveals the functional relationship (for small amplitude motion) of the two dynamic terms with respect to angle of attack and sideslip. In general, it is expected that a nonlinear relationship exists for the dynamic terms as functions of angle of attack and sideslip. The small amplitude analysis captures the locally linearized model variation with angle of attack and sideslip.

Step 2. In the second series of dynamic tests, a nonlinear model methodology may be required. These dynamic tests begin with single-frequency, one-degree-of-freedom, large-amplitude motions with constant nonzero sideslip during longitudinal motions and nonzero mean sideslip during lateral-directional motions over a large range of angle of attack and sideslip. With the assumption of small amplitude motion removed, the possibility of nonlinear response is more likely. As in the previous step, the simplifying assumption of superposition is still used so 
longitudinal and lateral axes are treated independently. Although each axis, individually, may exhibit nonlinear response this assumption is equivalent to assuming that no aerodynamic coupling exists between axes. Again harmonic analysis is applied to determine the complexity of the aerodynamic response. The existence of higher order harmonics directly indicates the presence of nonlinear response in the model, although it does not indicate which terms are contributing to the nonlinear response. However, identification is possible since the static term is completely known from direct tunnel measurements and the unsteady term can be separately estimated using oscillatory coning experiments ${ }^{16,20}$ or through planar oscillation experiments ${ }^{21}$ in heave and sway. The steady-flow damping term can be estimated knowing the other two terms or by using a curved-flow tunnel ${ }^{21,22}$ the term can be directly measured.

A constraint for this study is that only conventional single-frequency, one-degree-of-freedom, forced-oscillation test data are considered. Under this constraint some analysis is still possible. If the static and steady-flow damping terms are assumed to be nonlinear and well represented by higher order polynomials, then the order determined by harmonic analysis also implies the order of the polynomial required. For example, consider a conventional harmonic model for rolling moment analysis

$$
\Delta C_{\ell}=C_{\ell \beta} \beta+\frac{l}{V} C_{\ell \dot{\beta}} \dot{\beta}+\frac{l}{V} C_{\ell p} p+\left(\frac{l}{V}\right)^{2} C_{\ell \dot{p}} \dot{p}
$$

In this case, assuming $\phi=\phi_{A} \sin \omega t$ and $\beta=\phi \sin \theta$, it follows that a $1^{\text {st }}$ order harmonic model is

$$
\Delta C_{\ell}=\phi_{A}\left(C_{\ell \beta} \sin \theta-k^{2} C_{\ell \dot{p}}\right) \sin \omega t+\phi_{A} k\left(C_{\ell p}+C_{\ell \dot{\beta}} \sin \theta\right) \cos \omega t
$$

If a cubic nonlinearity exists in the static term, such that Eq. (21) contains the term $C_{\ell \beta^{3}} \beta^{3}$, then this term produces a cubic-harmonic sine term. Using the identity, $\sin ^{3} \omega t=1 / 4[3 \sin \omega t-\sin 3 \omega t]$, it can be shown that both $1^{\text {st }}$ and $3^{\text {rd }}$ harmonic terms are added to the model to produce

$$
\begin{gathered}
\Delta C_{\ell}=\phi_{A}\left(C_{\ell \beta} \sin \theta-k^{2} C_{\ell \dot{p}}+(3 / 4) \phi_{A}^{2} C_{\ell}{ }_{\beta^{3}} \sin ^{3} \theta\right) \sin \omega t \\
+\phi_{A} k\left(C_{\ell p}+C_{\ell \dot{\beta}} \sin \theta\right) \cos \omega t \\
-(1 / 4) \phi_{A}^{3} C_{\ell}{ }_{\beta^{3}} \sin ^{3} \theta \sin 3 \omega t
\end{gathered} .
$$

Eq. (23) shows that the cubic nonlinearity expresses itself as both a $3^{\text {rd }}$ order harmonic and an additional component added to the $1^{\text {st }}$ harmonic term. A similar analysis can be done for harmonics of different order and the same analysis can be applied to the steady-flow damping term. Unfortunately, the unsteady term does not lend itself to this type of analysis. Thus, the presence of a $3^{\text {rd }}$ order term in the static data or in the steady-flow damping term will result in a $3^{\text {rd }}$ harmonic during a general harmonic analysis, however, to determine the source of the higher harmonic response independent information about each of the three model terms is required.

One possible approach to the identification problem is to assume that the steady-flow damping term is known approximately from Step 1. Given this approximation and the static measurements, identification of the unsteady term and the steady-flow damping term can proceed using a relaxation method ${ }^{14}$. Since motions are limited (in this step) to one degree of freedom, forced-oscillations, a simplified version of model Eq. (11) can be used for model structure determination and parameter estimation. For examples in this paper, during large amplitude, body-axis roll oscillations $\left(\phi_{A} \leq 30^{\circ}\right.$, ) with a zero sideslip offset angle, the angle of attack will vary by less than 4 degrees. Even with a 15 degree mean offset in sideslip, the maximum angle of attack variation during oscillations is limited. Oscillations in $\alpha$ and $\beta$, during roll oscillations, vary according to the relations 


$$
\begin{aligned}
& \beta=\sin ^{-1}\left\{\sin (\phi) \sin \left(\theta_{0}\right) \cos \left(\psi_{0}\right)-\cos (\phi) \sin \left(\psi_{0}\right)\right\} \\
& \alpha=\tan ^{-1}\left\{\cos (\phi) \tan \left(\theta_{0}\right)+\frac{\sin (\phi) \sin \left(\psi_{0}\right)}{\cos \left(\theta_{0}\right) \cos \left(\psi_{0}\right)}\right\}
\end{aligned}
$$

where $\theta_{0}$ and $\psi_{0}$ are the nominal pitch and yaw offsets, respectively. In either example for this paper, it is reasonable to assume that $C_{\ell}$ can be expressed as $C_{\ell}(\beta, p)$ and that Eq. (11) can be written in simpler form as

$$
\begin{aligned}
& \dot{\eta}(t)=-b(\beta) \eta(t)-a(\beta) \dot{\beta} \\
& C_{a}(t)=C_{a}(\infty ; \beta)+\frac{l}{V} C_{a_{p}}(\infty ; \beta) p(t)+\eta(t)
\end{aligned}
$$

after substituting $\eta_{\beta}(t)$ for the deficiency function (and dropping the subscript $\beta$ ). A broad class of nonlinear unsteady responses can be modeled with this model structure where the static term, steady-flow damping term, and indicial term parameters can vary nonlinearly with $\beta$. The unsteady term has the form of a linear parameter varying (LPV) system ${ }^{23}$ where the parameter is the state, $\beta$, instead of a general parameter. In this step, it is assumed that each of the four unknown parameters $\left(C_{\ell}(\infty ; \beta), C_{\ell p}(\infty ; \beta), a(\beta), b(\beta)\right)$ can be represented by polynomials in $\beta$.

The identification problem for the model in Eq. (25) is solved in a two stage iterative process, shown in Fig. 1. First model structure determination of the unsteady term polynomials, $a(\beta)$ and $b(\beta)$, is performed using stepwise regression $^{2}$ (SR). To setup the regression equation, a variable $y(t)$ is formed by subtracting the static term and approximated steady-flow damping term from the force or moment measurement, $C_{a}(t)$, as

$$
y(t)=C_{a}(t)-C_{a}(\infty ; \beta)-\frac{l}{V} C_{a p}(\infty ; \beta) p(t)
$$

Then the regression equation, showing the discrete measured values at time $t(i)$, is given as

$$
\dot{y}_{E}(i)=-\left[b\left(\beta_{E}(i)\right) \eta_{E}(i)+a\left(\beta_{E}(i)\right) \dot{\beta}_{E}(i)\right]+\varepsilon_{\eta}(i)
$$

where index $E$ indicates the measured values and $\varepsilon_{\eta}(i)$ is an equation error. Derivatives of measurement, $y_{E}$, are formed using a locally smoothed numerical derivative $e^{24,25}$. Parameter estimates are then updated with Maximum likelihood (ML) estimation. State and measurement equations for ML estimation are

$$
\begin{aligned}
& \dot{\eta}(t)=-b(\beta) \eta(t)-a(\beta) \dot{\beta}_{E} ; \quad \eta(t=0)=\eta(0) \\
& \eta_{E}(t)=C_{a_{E}}(i)-C_{a}\left(\infty ; \beta_{E}(i)\right)-\frac{l}{V} C_{a_{p}}\left(\infty ; \beta_{E}(i)\right) p_{E}(i)+v(i), \quad i=1,2, \ldots, N
\end{aligned}
$$

A second stage identification is done since $C_{a_{p}}(\infty ; \beta)$ is only known approximately and the estimates of $a(\beta)$ and $b(\beta)$ are based on this a priori information. To update the damping term, a second variable is formed

$$
\begin{aligned}
z(t) & =C_{a}(t)-C_{a}(\infty ; \beta)-\eta(t) \\
& =\frac{l}{V} C_{a_{p}}(\infty ; \beta) p(t)
\end{aligned}
$$


If the measured values and new estimates for the unsteady term are substituted into Eq. (29), a new regression equation is obtained as

$$
z_{E}(i)=\frac{l}{V} C_{a_{p}}\left(\infty ; \beta_{E}(i)\right) p_{E}(i)+\varepsilon_{z}(i), \quad i=1,2, \ldots, n
$$

The model structure and parameters for the steady-flow damping term can be estimated using Eq. (30). This completes the first iteration of the two stage identification. In the next iteration, $a(\beta)$ and $b(\beta)$ can be estimated again using the new values and model structure for the steady-flow damping term. This process is continued until parameter estimates have converged.

Step 3. In the last series of dynamic tests, a nonlinear model methodology is required. The last assumption to be removed is that superposition of orthogonal planar motions is valid. For this case experiments must be designed to produce coupling motions that can be used to test the superposition assumption. If this assumption is proved false then experiments designed to allow simultaneous estimation of both longitudinal and lateral-directional general model terms are required. From Ref. 3 a set of four experiments are suggested for the case where linear coupling occurs and under conditions of nonlinear coupling more complex experiments requiring oscillatory coning may be required. These types of experiments were not available for this study.

\section{Experiments}

Dynamic tests were conducted with a $2.5 \%$ scale model of the F-16XL (Fig. 2) in the water tunnel at Rolling Hills Research Company to identify nonlinear aerodynamic model parameters. For these tests the scale model was mounted on a dynamic test rig through a five-component strain-gauge balance (axial force was not measured). The dynamic test rig is a computer-controlled system with a sting-mounted double C-strut support system ${ }^{26}$. The mounting arrangement rotated the model about the reference center of gravity location of $0.558 \bar{c}$. The tests were conducted at a dynamic pressure of $0.81 \mathrm{psf}$ resulting in the flow velocity of 11 inches/sec and a Reynolds number of $52 \times 10^{3}$ based on the mean aerodynamic chord. Reynolds number values different from that found in flight or wind tunnels are acceptable for this study since the data are used to both estimate and predict responses under the same conditions. With this relaxed requirement, hydrodynamic flow at the inlet was improved by adding an inlet fairing to block flow into the inlet. Flow visualization and measurement equipment inside the model did not allow enough space for smooth flow through the body. All data were obtained with the leading edge flaps and trailingedge surfaces at $0^{\circ}$ deflection.

Although forced oscillation tests were performed about all three body axes, only roll axis oscillation data are considered in this paper. Single-frequency and wide-band (Schroeder sweeps ${ }^{15,17}$ ) forced-oscillation tests were performed about 14 mean angles of attack, $\alpha_{0}=[10,15,20,25,30,32.5,35,37.5,40,42.5,45,47.5,50,55,60]$,

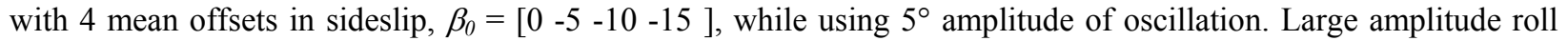
cases were done at the same $\alpha_{0}$, for a reduced set of offsets, $\beta_{0}=\left[\begin{array}{ll}0 & -5\end{array}\right]$, and amplitudes of $\left[\begin{array}{llll}10 & 15 & 20 & 30\end{array}\right]$ degrees. Five non-dimensional frequencies, $\mathrm{k}=[0.066,0.131,0.197,0.262,0.328]$, were used for small amplitude tests and six non-dimensional frequencies, $\mathrm{k}=[0.066,0.095,0.131,0.160,0.197,0.262]$, were used for large amplitude roll oscillation tests. Data were sampled at $10 \mathrm{~Hz}$ with a low pass analog filter at $5 \mathrm{~Hz}$. For these experiments 30 cycles of data were recorded for each run and then an averaged cycle was formed to minimize measurement noise. The averaged measurements were used for small amplitude analysis and 3 cycles of measured data were used for the large amplitude analysis. Static data were obtained in $1^{\circ}$ increments for angles of attack from $0^{\circ}$ to $70^{\circ}$ and for sideslip angle from $-20^{\circ}$ to $20^{\circ}$.

\section{Results and Discussion}

Figure 3a presents the static data for $C_{\ell}(\infty ; \alpha, \beta)$ as a surface plot and Fig. $3 \mathrm{~b}$ shows the corresponding contour plot. This plot is representative of all the aerodynamic static data in that the surface is relatively smooth and linear at low and very high angles of attack. In the mid angle-of-attack range, $20^{\circ} \leq \alpha \leq 50^{\circ}$, fairly severe static nonlinearities occur and the model is slightly asymmetric. This may limit the effectiveness of a simple polynomial representation of the underlying static nonlinearities as part of the overall model. 
Harmonic analysis of the small-amplitude oscillatory data was performed on single oscillations formed by averaging 30 cycles of data. This analysis was done for each of the 5 frequencies. Figure 4 shows representative input / output data used in this analysis. The figure shows averaged small-amplitude roll oscillation time histories for $\beta(t), p(t)$, and $C_{\ell}(t ; \alpha(t), \beta(t))$ at a low and high frequency with offset $\beta_{0}=0^{\circ}$ and $\alpha_{0}=30^{\circ}$. With small-amplitude oscillations a linear model is generally expected to be adequate for all $\alpha_{0}$ and $\beta_{0}$ set points. Harmonic analysis confirmed this for most conditions tested, however, at $\alpha_{0}=42.5^{\circ}, \beta_{0}=0^{\circ}, \mathrm{k}=0.066$, and at $\alpha_{0}=\left[37.5^{\circ}, 40^{\circ}\right]$, with $\beta_{0}=-5^{\circ}$, and $\mathrm{k}=0.066$, the Multiple Correlation Coefficient, $\mathrm{R}^{2}$ was relatively low (less than 0.6 ), indicating less adequate linear models for these conditions.

Analysis of the small amplitude data provides a basis for the nonlinear analysis. The variation of linear model terms with angle of attack and sideslip defines the initial model structure used in the nonlinear analysis. First-order harmonic analysis provided in-phase and out-of-phase components, $\left(\bar{C}_{\ell \beta}, \bar{C}_{\ell p}\right)$. Out-of-phase $\left(\bar{C}_{\ell p}\right)$, damping terms are shown in Fig. 5 over the range of angle of attack, frequencies, and $\beta_{0}$ offsets. These results show a strong frequency dependence or unsteady behavior for $25^{\circ}<\alpha<40^{\circ}$, with $\beta_{0}=0^{\circ}$, and for $25^{\circ}<\alpha<37.5^{\circ}$, with $\beta_{0}=-5^{\circ}$. For offsets of $\beta_{0}=\left[-10^{\circ},-15^{\circ}\right]$ the unsteady behavior is practically nonexistent. Overall a linear model is reasonable for the small amplitude data, thus Two-Step Regression ${ }^{10,27}$ was applied to the in-phase and out-of-phase data to produce a linear unsteady model where appropriate. As an example, Fig. 6 highlights the linear model parameters $\left(C_{\ell \beta}(\infty), C_{\ell p}(\infty), a, \tau_{l}\right)$ over the range of angle of attack for $\beta_{0}=0$. At $\alpha_{0}=42.5^{\circ}$, the parameter estimates are in doubt due to poor $\mathrm{R}^{2}$ value. For this example, $C_{\ell \beta}(\infty)$, estimated by regression matches well with the slopes estimated directly from the static data indicating relatively good identification. The steady-flow damping term, $C_{\ell p}(\infty)$, is relatively near -0.2 , except at $\alpha_{0}=\left[35^{\circ}, 37.5^{\circ}\right]$ where it moves closer to 0 . The unsteady term gain, $a / b$, confirms that for $25^{\circ}<\alpha<40^{\circ}$, unsteady behavior is present. The gain values become very small outside of this range of angle of attack indicating no significant unsteady behavior. The non-dimensional time constant, $\tau_{l}$, has a maximum value of 20 in the unsteady region and values less than 10 outside this region. The lack of unsteady behavior at $\alpha_{0}=50^{\circ}$ resulted in a poor $\tau_{1}$ estimate with large error bounds. Figure 7 summarizes $C_{\ell p}(\infty)$ for each $\beta_{0}$ offset at $\alpha_{0}$ $=\left[30^{\circ}, 37.5^{\circ}\right]$. For $\alpha_{0}=37.5^{\circ}, \beta_{0}=-5^{\circ}, C_{\ell p}(\infty)$ is rejected due to poor $\mathrm{R}^{2}$ and the lack of consistent frequency distribution in the harmonic coefficients. These estimates have large error bounds reflecting the lack of information content. The lack of unsteady behavior and inconsistent frequency distribution in this range can be seen in Fig. 5 and Fig. 8. A restriction for the linear model in Eq. (14) is that it only provides an adequate model for portions of the envelope where unsteady behavior occurs, as indicated by region II in Fig. 8.

Figures $9 \mathrm{a}$ and $9 \mathrm{~b}$ show sample time histories of the large amplitude oscillatory data at $\alpha_{0}=30^{\circ}$ and $37.5^{\circ}$, each case with $\beta_{0}=0^{\circ}$. The figures show four cycles of oscillation for each of the four frequency cases used in analysis, $\mathrm{k}=[0.066$, $0.095,0.131,0.160]$. The time histories have been

Table I. Linear model parameter estimates and standard errors (in parentheses) for $C_{\ell}$, using two identification methods, at $\alpha_{0}=30^{\circ}$, $\beta_{0}=\mathbf{0}^{\circ}, \phi_{A}=3^{\circ}$.

\begin{tabular}{|c|c|c|}
\hline Parameter & $\begin{array}{c}\text { 2-Step } \\
\text { Analysis }\end{array}$ & $\begin{array}{c}\text { Relaxation } \\
\text { Method }\end{array}$ \\
\hline$C_{\ell \beta}(\infty)$ & 0.06 & 0.063 \\
& $(0.012)$ & $(\mathrm{n} / \mathrm{a})$ \\
\hline$C_{\ell p}(\infty)$ & -0.28 & -0.321 \\
& $(0.010)$ & $(0.0026)$ \\
\hline$a$ & 0.305 & 0.309 \\
& $(0.0049)$ & $(0.001)$ \\
\hline$b$ & 0.15 & 0.156 \\
& $(0.0025)$ & $(0.0009)$ \\
\hline$\tau_{l}$ & 14.2 & 14.5 \\
& $(0.24)$ & $(0.0020)$ \\
\hline $\mathrm{R}^{2}$ & 0.99 & 0.98 \\
\hline
\end{tabular}
concatenated to facilitate data handling. The first cycle of predicted response is eliminated to remove any initial transients and ensure that steady harmonic motion is used in calculating residuals. The amplitude, $\phi_{A}$, is the same for each case shown, however, the resulting $\alpha$ and $\beta$ oscillations change during roll oscillations according to Eq. (24). The distortion of the rolling moment response from a steady harmonic wave, in particular for the low frequency cases, indicates nonlinear behavior.

Harmonic analysis was applied to the large amplitude data. First in-phase and out-of-phase components were calculated from the $1^{\text {st }}$ harmonic coefficients. Plots of the out-of-phase components over the range of angle of attack and frequencies are shown in Fig. 10 with $\beta_{0}=0$. This graph shows the effect of oscillation amplitude on the damping coefficient relative to the small amplitude case shown in Fig. 5. For the large amplitude case the unsteady behavior is observed over approximately the same range of angle of attack $\left(25^{\circ}<\alpha<40^{\circ}\right)$ as the low amplitude case, however, the magnitude of the out-of-phase damping is reduced by almost half. Figure 11 shows the multiple 
correlation coefficient, $\mathrm{R}^{2}$, from the large amplitude analysis. The upper graphic shows the variation of $\mathrm{R}^{2}$ with angle of attack and non-dimensional frequency, $\mathrm{k}$. In this case the low frequency run with $\mathrm{k}=0.066$, has the lowest values of $\mathrm{R}^{2}$ for $35^{\circ} \leq \alpha \leq 40^{\circ}$, indicating some degradation in the linear model at these angles of attack. The lower graphic shows the variation of $\mathrm{R}^{2}$ with angle of attack and harmonic model order for this worst case frequency, $\mathrm{k}=0.066$. From the harmonic analysis, only adding a $3^{\text {rd }}$ order harmonic improved the result. One explanation of this could be the presence of a $3^{\text {rd }}$ order polynomial nonlinearity in either the static or damping term. In order to identify the model in this case the relaxation method is applied.

To demonstrate the method, two examples are considered using the data in Fig. 9. The first example, at $\alpha_{0}=30^{\circ}$, considers linear unsteady behavior that can be adequately modeled by the linear model methodology. Application of the relaxation method allows for more complex modeling but in this case it is used to confirm the linear analysis and validate the method. Parameter estimates and their standard errors using both methods are given in Table I. Both

Table II. Linear models for $C_{\ell}$ at $\alpha_{0}=37.5^{\circ}, \beta_{0}=0^{\circ}$.

\begin{tabular}{|c|c|c|}
\hline Parameter & $\begin{array}{c}\text { Linear Model } \\
\boldsymbol{\phi}_{\boldsymbol{A}}=\mathbf{5}^{\circ}\end{array}$ & $\begin{array}{c}\text { Linear Model } \\
\boldsymbol{\phi}_{A}=\mathbf{3 0}^{\circ}\end{array}$ \\
\hline$C_{\ell \beta}(\infty)$ & 0.15 & -0.015 \\
& $(0.045)$ & $(0.0096)$ \\
\hline$C_{\ell p}(\infty ; \beta)$ & -0.13 & -0.31 \\
& $(0.049)$ & $(0.020)$ \\
\hline$a(\beta)$ & 0.47 & 0.242 \\
& $(0.023)$ & $(0.0095)$ \\
\hline$b(\beta)$ & 0.28 & 0.44 \\
& $(0.032)$ & $(0.035)$ \\
\hline$\tau_{1}(\beta)$ & 8.0 & 5.1 \\
& $(0.90)$ & $(0.41)$ \\
\hline $\mathrm{R}^{2}$ & $0.90(\mathrm{k}=0.066)$ & $0.78(\mathrm{k}=0.066)$ \\
\hline
\end{tabular}
methods provide nominally the same models. Figure 12 shows a comparison of measured and predicted response, in a phase-plane plot, using the linear model obtained from the relaxation method. Further refinements are possible, such as modeling the static data, shown in Fig. 13, with a cubic or even $5^{\text {th }}$ order polynomial to more exactly capture the true nature of the system. However, in this case the static moment contribution is a factor of 10 smaller than the damping term contribution; consequently the increased model complexity is not justified. $\mathrm{R}^{2}$ values in Fig. 11 confirm this assumption for all frequencies.

Measurements at $\alpha_{0}=37.5^{\circ}$ provide an example of response with nonlinear behavior, based on $\mathrm{R}^{2}$ values shown in figure 11, and strong unsteady behavior based on frequency dependence of in-phase and out-of-phase coefficients shown in figure 10. Figure $9 \mathrm{~b}$ shows the responses at all frequencies are distorted from a linear sinusoidal response. Since the harmonic analysis inferred that at least a cubic nonlinearity is present and the static component is a relatively large component in this case, a cubic representation of the static data was initially implemented. It was found, however, that a $5^{\text {th }}$ order polynomial was required for an adequate static model. Static rolling-moment coefficient, $C_{\ell}\left(\infty ; \alpha_{0}=37.5, \beta\right)$, is presented in Fig. 14 together with its approximation by the $5^{\text {th }}$ order polynomial. Results from linear regression provided initial estimates for the damping and unsteady terms in the postulated nonlinear model. Table II shows linear models estimated from both small and large amplitude data. Linear analysis of the large amplitude data produced a slightly different model compared to the small amplitude case reflecting the amplitude dependence of nonlinear systems.

Table III. Nonlinear model for $C_{\ell}$ at $\alpha_{0}=37.5^{\circ}, \beta_{0}=0^{\circ}$.

\begin{tabular}{|c|c|}
\hline Parameter & Nonlinear Model \\
\hline$C_{\ell}(\infty ; \beta)$ & $0.007+0.317 \beta+0.202 \beta^{2}-12.786 \beta^{3}$ \\
& $-2.371 \beta^{4}+91.123 \beta^{5}$ \\
\hline$C_{\ell p}(\infty ; \beta)$ & $-0.786-1.791 \beta^{2}+97.616 \beta^{4}$ \\
& $(0.0045)(0.34)(4.48)$ \\
\hline$a(\beta)$ & $0.527-43.668 \beta^{2}-507.228 \beta^{4}$ \\
& $(0.0051)(0.39)(5.096)$ \\
\hline$b(\beta)$ & $0.624-8.109 \beta^{2}$ \\
& $(0.011)(0.14)$ \\
\hline$\tau_{1}(\beta=0)$ & 3.63 \\
& $(0.027)$ \\
\hline $\mathrm{R}^{2}$ & 0.97 \\
\hline
\end{tabular}

Estimated parameters are shown with their

standard errors below the estimate in parentheses. Table III shows the final estimated model from the relaxation method applied at $\alpha_{0}=37.5^{\circ}$ and the corresponding overall $\mathrm{R}^{2}$ for all four frequencies. The parameter covariance matrix for this case contained some large pair-wise correlations indicating identifiability may be a problem. Figure 15 shows the measured and predicted rolling moments for the four frequencies considered. These graphs all show distortion from the regular elliptic shape associated with linear response. For each frequency, the nonlinear model prediction matches the response well. At the lowest frequency, $\mathrm{k}=0.066$, the distortion or nonlinearity is greatest, but the model match is still very good. Although the fit is very good the model is not completely satisfactory due to 
the inconsistency with the small-amplitude linear analysis shown in Table II. If the nonlinear model can represent the linear case as a subset then, for example, at $\beta=0$, the nonlinear model estimate, $C_{\ell p}(\infty ; \beta=0)$, should be close

to the corresponding linear model analysis of the small amplitude data, a value of -0.13 . The differences may reflect a number of possible sources of error besides the model structure for the damping and unsteady terms, such as data accuracy and information content of the data.

\section{Concluding Remarks}

This paper introduces a general formulation of the aerodynamic model for aircraft that includes nonlinear unsteady aerodynamics and suggests a progressive series of steps toward development of a nonlinear model identification methodology that defines the mathematical model, algorithms for identification, and required test techniques. The mathematical model presented can predict a broad class of aerodynamic responses of rigid-body aircraft. An important element of the methodology is the form used for the aerodynamic model. This form retains conventional static and rotary aerodynamic terms that have traditionally provided substantial engineering information to the flight dynamics community. This structure allows easy interpretation of the model parameters by retaining conventional stability and control derivatives for static and dynamic terms. Unsteady terms in the form of indicial functions are added to expand the model structure and allow frequency dependent phenomena to be modeled. In the nonlinear case, parameters in each of these terms may vary with angle of attack and sideslip. Thus, static and rotary dynamic terms can be represented as polynomials or splines and the indicial term as a linear parameter varying system. This approach produces a straightforward and efficient model structure for simulation and design since these models are more general than conventional locally-linearized models but still easily incorporated into conventional table look up formats.

As a practical matter, it is desirable to develop an adequate model that represents the physics of interest without unnecessary complexity. In addition, the mathematical model must lend itself to identification from experimental data. This suggests model development should start with the least complicated model and add complexity only as needed. Initial steps for developing a nonlinear model identification methodology with this philosophy are applied to static and forced oscillation data to identify rolling moment coefficient. For nonlinear model identification a twostage identification procedure was used. From experimental data it was assumed that the static and rotary-dynamic terms were known. The method combined stepwise regression and maximum likelihood estimation to determine model structure and parameter estimates for the indicial term. When a priori information about the rotary term is poor, a two-stage optimization algorithm can be used to identify both the unsteady and rotary terms. The method was applied to a linear unsteady case that validated the approach against a 2-Step Regression method. A second case considered a nonlinear unsteady model that provided good fit to the nonlinear dynamic response measurements. The nonlinear model was not completely satisfactory, however, because it did not reduce to the linear model for smallamplitude motions. High correlations for some parameter estimates suggest an identifiability issue that requires further review of the methodology, experiment design, and measurement accuracy. Nonlinear modeling experiments, in particular, require careful attention to these issues. An assumption in the proposed candidate methodology that new measurements could be formed by differencing the measured rolling moment with the static and damping terms to form a new indicial measurement was not validated by this study. This approach assumes that the new measurement will be corrupted primarily by measurement noise and not model error. The successful application of this assumption depended on the relative magnitudes of the three terms in the model.

Further development of nonlinear model identification methodology is needed to realize the capability for predicting aircraft aerodynamic response under arbitrary motion. Future work should refine experiment design and identification algorithms, and investigate a range of input amplitudes and wide-band inputs for maximizing information content and testing efficiency. Advanced test techniques such as oscillatory coning, and planar forcedoscillation in heave and sway should be studied since these offer a direct measurement of unsteady acceleration terms and can provide validation of the nonlinear model identification methodology.

\section{References}

\footnotetext{
${ }^{1}$ Byran, G. H.: Stability in Aviation, MacMillan and Co., London, 1911.

${ }^{2}$ Klein, V., Batterson, J.G., Murphy, P.C.: Determination of Airplane Model Structure from Flight Data by Using Modified Stepwise Regression, NASA TP 1916, 1981.

${ }^{3}$ Tobak and Schiff: On the Formulation of the Aerodynamic Characteristics in Aircraft Dynamics, NASA TR R-456, 1976.
} 
${ }^{4}$ Goman, M.G., Stolyaraov, G. I., Tyrtyshnikov, S.L., Usolcev, S.P., and Khrabrov, A.N.: Mathematical Description of Longitudinal Aerodynamic Characteristics at High Angles of Attack Accounting for Dynamic Effects of Separated Flow. TsAGI Preprint No. 9, 1990 (in Russian).

${ }^{5}$ Goman, M. and Khrabrov, A.(TsAGI): State-Space Representation of Aerodynamic Characteristics of an Aircraft at High Angles of Attack. Journal of Aircraft, Vol.31, No.5, 1994, pp. 1109-1115.

${ }^{6}$ Goman, M., Khrabrov, A., and Usolcev, S.: Unsteady Aerodynamic Model for Large Amplitude Maneuvers and its Parameter Identification. $11^{\text {th }}$ IFAC Symposium on Identification, July 8-11, 1997, Kitakyushu, Japan, Vol. 1, pp.399-403.

${ }^{7}$ Fishenberg, D.: Identification of an Unsteady Aerodynamic Stall Model from Flight Test Data. AIAA Paper 95-3438-P, 1996.

${ }^{8}$ Klein, V., and Noderer, K.D.: Modeling of Aircraft Unsteady Aerodynamic Characteristics”, Part I - Postulated Models, NASA TM 109120, May, 1994; Part 2 - Parameters Estimated From Wind Tunnel Data, NASA TM 110161, April 1995; Part 3 Parameters Estimated From Flight Data, NASA TM 110259, May 1996.

${ }^{9}$ Klein, V., Murphy, P.C., Curry, T.J., and Brandon, J.M.: Analysis of Wind Tunnel Longitudianl Static and Oscillatory Data of the F-16XL Aircraft, NASA/TM-97-206276, December 1997.

${ }^{10}$ Klein, V., Murphy, P.C., and Szyba, N.M.: Analysis of Wind Tunnel Lateral Oscillatory Data of the F-16XL Aircraft, NASA/TM-2004-213246, July 2004.

${ }^{11}$ Myatt, James, H.: Modeling the Rolling 65-Degree Delta Wing with Critical State Encounters, AIAA 97-3646, August 1997.

${ }^{12}$ Chin, S., and Lan, E.: Fourier Functional Analysis for Unsteady Aerodynamic Modeling. AIAA Paper 91-2867-CP, 1991.

${ }^{13}$ Reisenthel, P. H.: Application of Nonlinear Indicial Modeling to the Prediction of a Dynamically Stalling Wing. AIAA 962493, August, 1996.

${ }^{14}$ Klein, V., Murphy, P.C.: Estimation of Aircraft Nonlinear Unsteady Parameters From Wind Tunnel Data, NASA TM-1998208969, December 1998.

${ }^{15}$ Murphy, P.C., Klein, V.: Estimation of Aircraft Unsteady Aerodynamic Parameters From Dynamic Wind Tunnel Testing, AIAA Paper 2001-4016, August 2001.

${ }^{16}$ Murphy, P.C., Klein, V.: Validation of Methodology for Estimating Aircraft Unsteady Aerodynamic Parameters from Dynamic Wind Tunnel Tests, AIAA 2003-5397, August, 2003.

${ }^{17}$ Schroeder, M.R.: Synthesis of Low-Peak-Factor Signals and Binary Sequences with Low Autocorrelation, IEEE Transactions on Information Theory, January 1970, pp. 85-89.

${ }^{18}$ Morelli, Eugene A.: High Accuracy Evaluation of the Finite Fourier Transform Using Sampled Data, NASA TM 110340 , June 1997.

${ }^{19}$ Kim, S., Murphy, P.C., Klein, V.: Evaluation and Analysis of F-16XL Wind Tunnel Data from Static and Dynamic Tests, NASA TM 213234, June, 2004.

${ }^{20}$ Khrabrov, A., Kolinko, K., Miatov, O., Vinogradov, J., Zhuk, A.: Using of Oscillatory Conning Experimental Rig for Separation of Rotary and Unsteady Aerodynamic Dervivatives, $18^{\text {th }}$ ICIASF, Toulouse, France, June 14-17, 1999

${ }^{21}$ Chambers, J.R., Grafton, Sue B., and Lutze, F.H.: Curved-Flow, Rolling-Flow, and Oscillatory Pure-Yawing Wind-Tunnel Test Methods for Determination of Dynamic Stability Derivatives, AGARD Lecture Series No. 114, Dynamic Stability Parameters, Athens, 1979, pp. 7.1-7.13.

${ }^{22}$ Lutze, F.H.: Experimental Determination of Pure Rotary Stability Derivatives Using a Curved and Rolling Flow Wind Tunnel, AIAA 1980-0309, January 1980.

${ }^{23}$ Marcos, A., Balas, G.: Linear Parameter Varying Modeling of the Boeing 747-100/200 Longitudinal Motion, AIAA 20014347, August 2001.

${ }^{24}$ Morelli, E.A.: System Identification Programs for Aircraft (SIDPAC), AIAA 2002-4704, August 2002.

${ }^{25}$ Lanczos, C.: Applied Analysis, Dover Publications, Inc., New York, ISBN 0-486-65655-X, 1988.

${ }^{26}$ Eidetics Corporation: Final Report; Determination of Nonlinear Dynamic Aerodynamic Coefficients for Aircraft, SBIR Phase II Final Report, Naval Air Warfare Center Aircraft Division, TR00-002, June 11, 2000.

${ }^{27}$ Abramov, N. B., Goman, M. G., Greenwell, D. J., and Khrabrov, A. N.: Two-Step Linear Regression for Identification of High Incidence Unsteady Aerodynamic Model. AIAA Paper 2001-4080, 2001. 


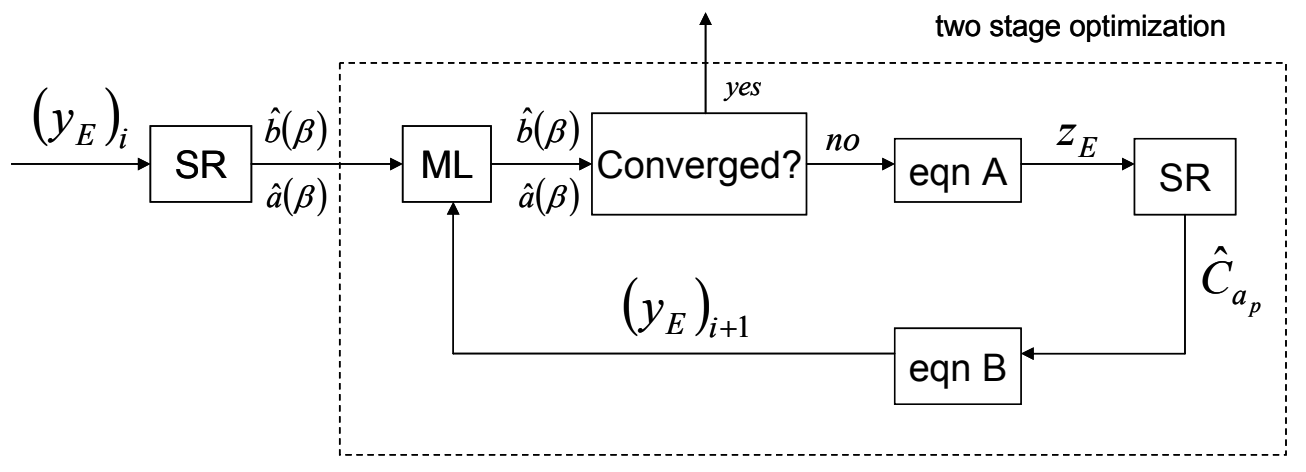

Eqn. A: $z_{E}(t)=\left(\frac{\ell}{V}\right) C_{a_{p}}\left(\infty ; \alpha_{0}, \beta\right) \mathrm{p}(t)$

Eqn. B: $\quad y_{E}(t)=C_{a}(t)-C_{a}\left(\infty ; \alpha_{0}, \beta\right)-\left(\frac{\ell}{V}\right) C_{a_{p}}\left(\infty ; \alpha_{0}, \beta\right) \mathrm{p}(t)$

Figure 1. Block diagram of model identification procedure using stepwise regression (SR) and a maximum likelihood (ML) estimation.

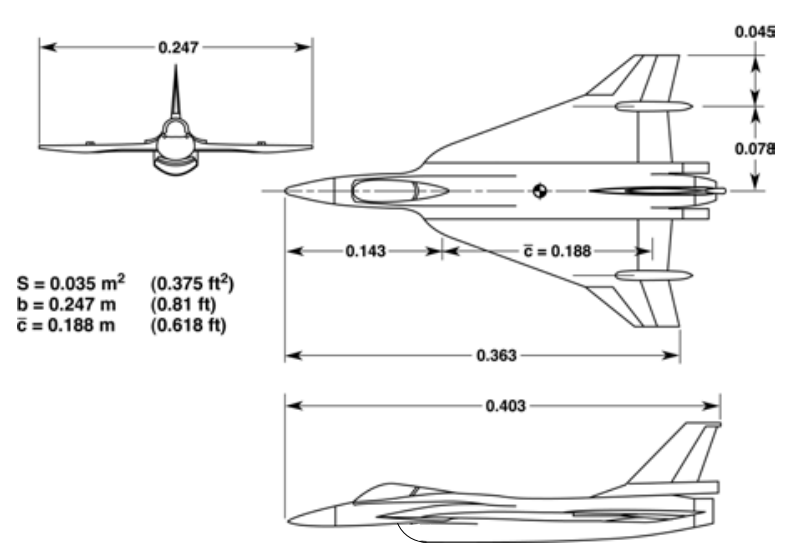

Figure 2. Three-view drawing of 2.5\% F-16XL model.

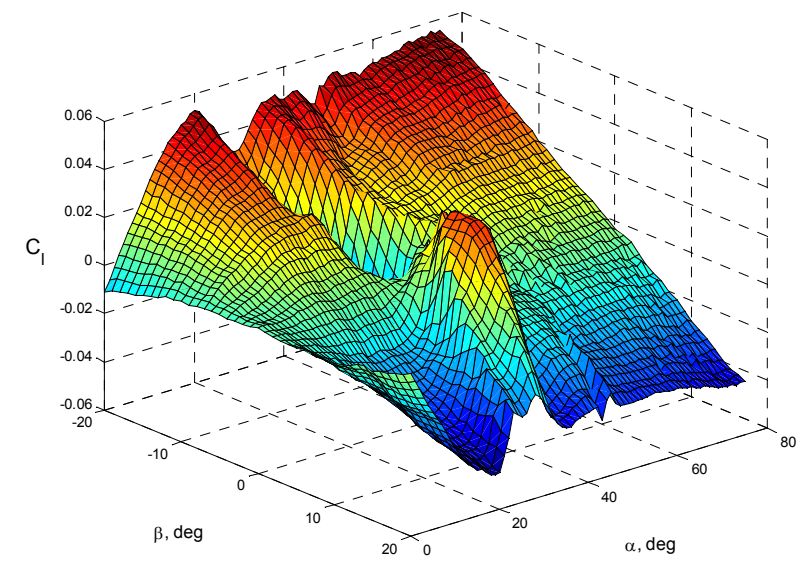

Figure 3a. Surface plot of $C_{\ell}(\infty ; \alpha, \beta)$. 


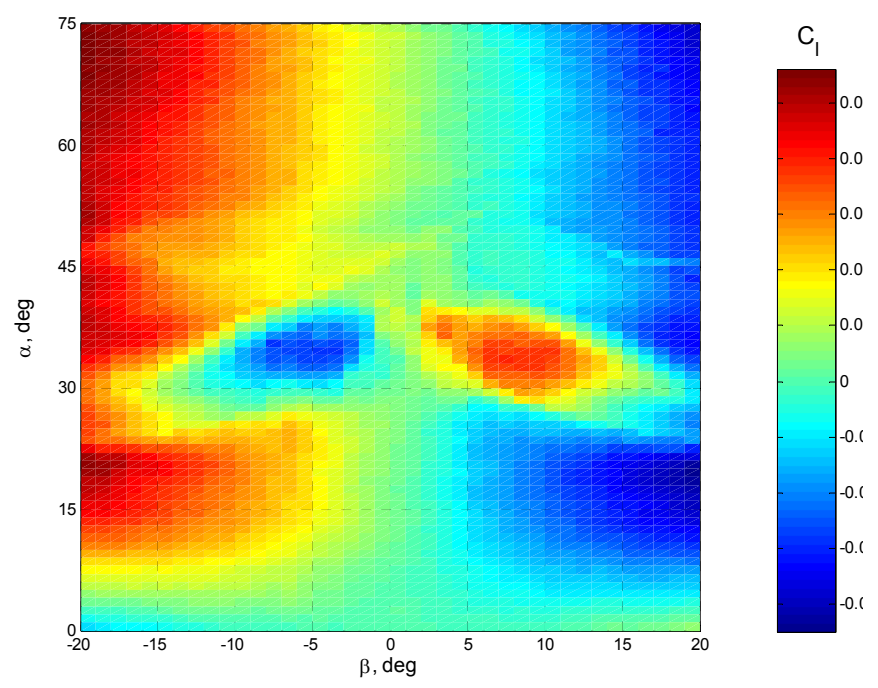

Figure 3b. Contour plot of $C_{\ell}(\infty ; \alpha, \beta)$.
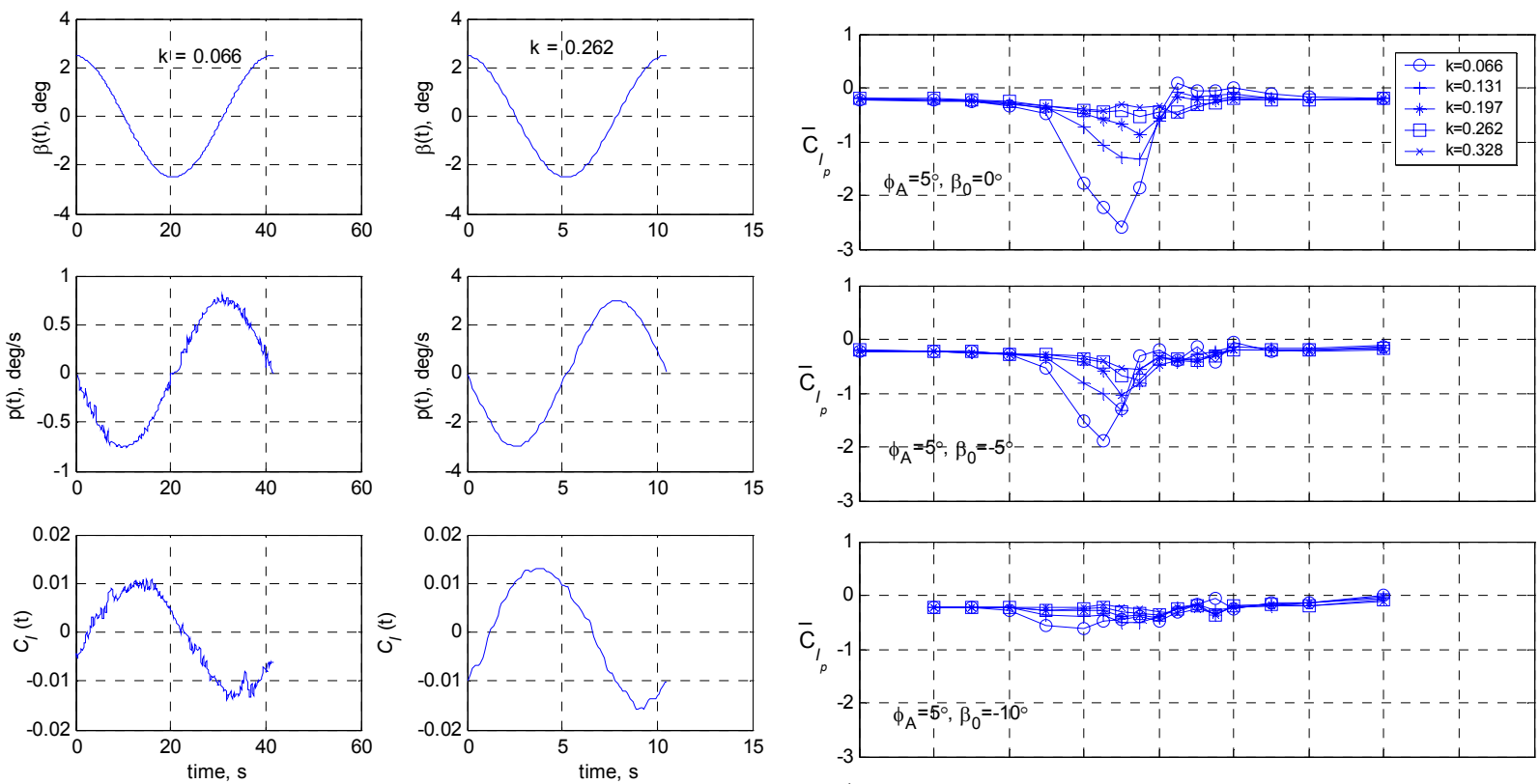

Figure 4. Average 1-cycle time histories of $\beta, p$, and $C_{\ell}$ from oscillatory tests at two frequencies. $\phi_{A}=5^{\circ}, \alpha_{0}=$ $30^{\circ}, \beta_{0}=0^{\circ}$.

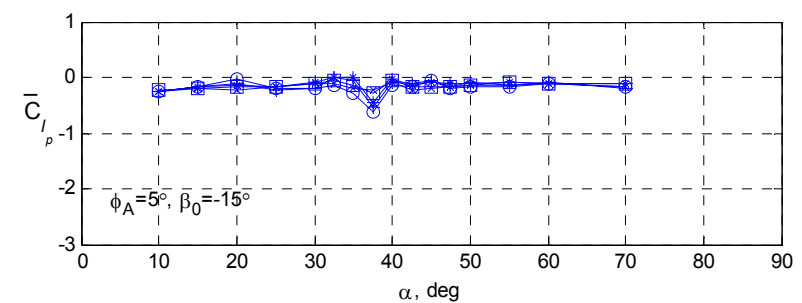

Figure 5. Variation of out-of-phase component with angle of attack, offsets in sideslip, and frequency of roll oscillations. 

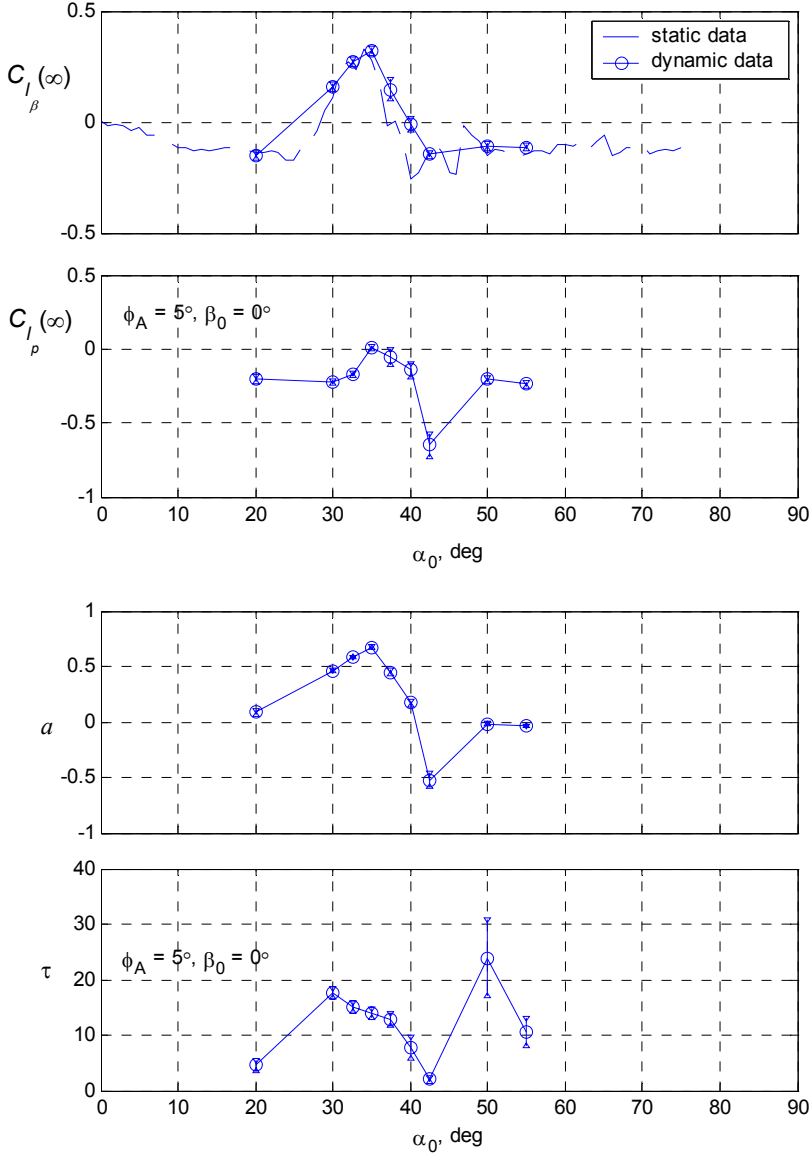

Figure 6. Estimated linear model parameters for rollingmoment coefficient using 2-Step Regression.

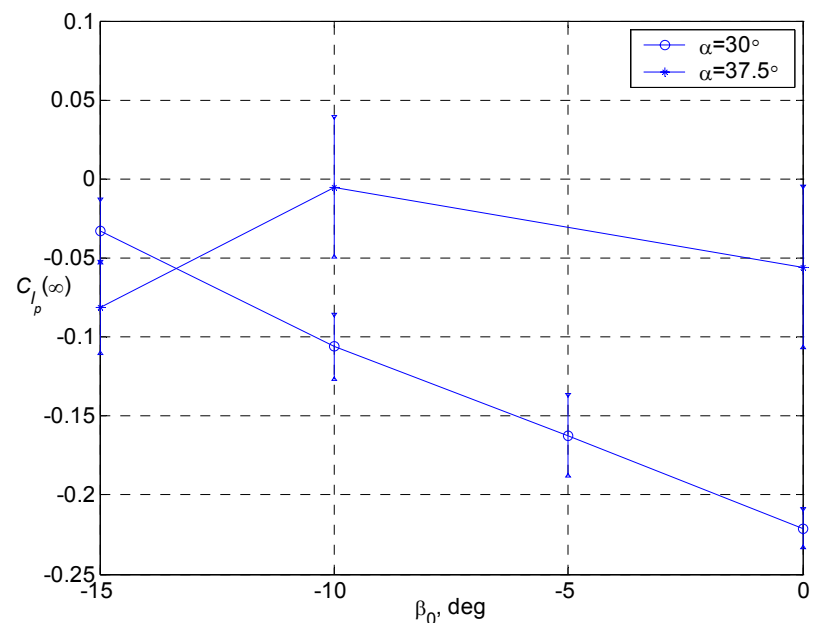

Figure 7. Variation of damping-in-roll parameter with sideslip angle at two values of angle of attack.

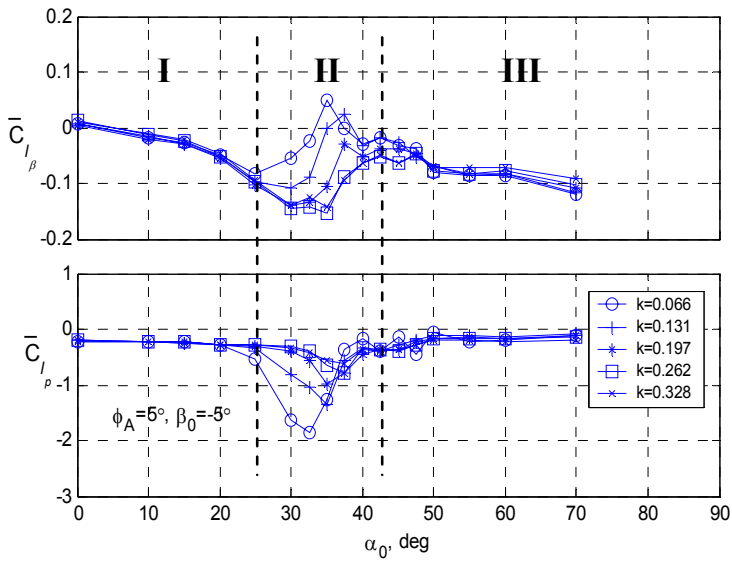

Figure 8. Three regions with different dynamics for roll moment in-phase and out-of-phase components. 


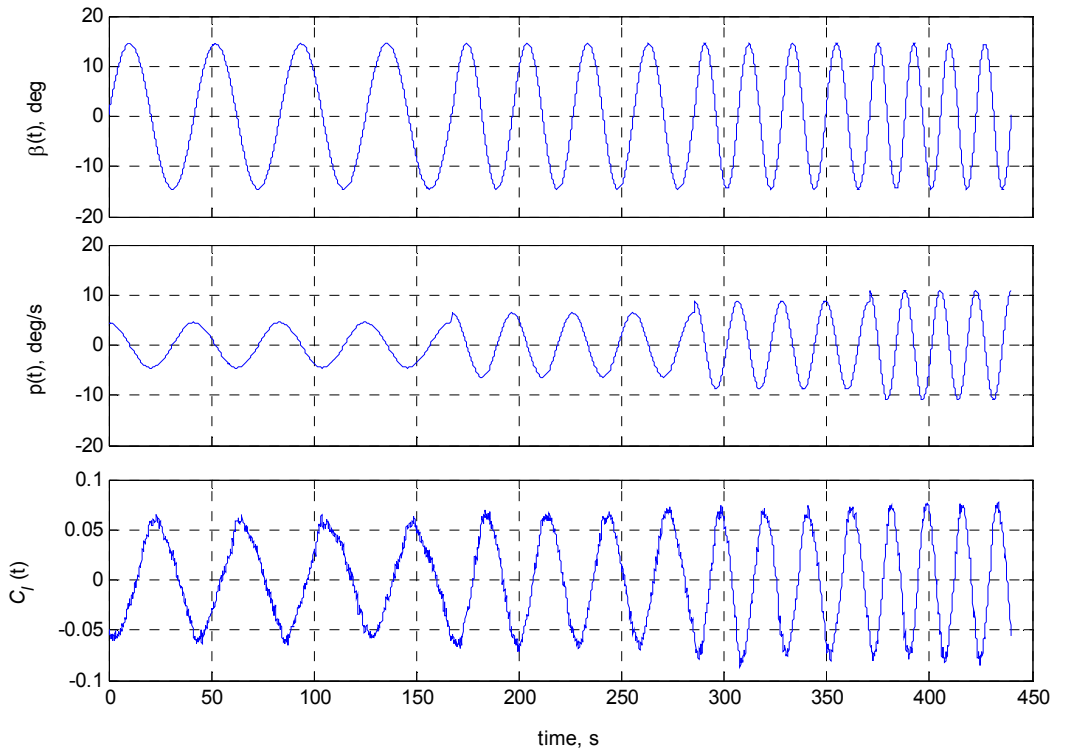

Figure 9a. Time histories of the large amplitude oscillatory data at $\alpha_{0}=30^{\circ}, \beta_{0}=$ $0^{\circ}$, and four frequencies, $k=[0.066,0.095,0.131,0.160]$.
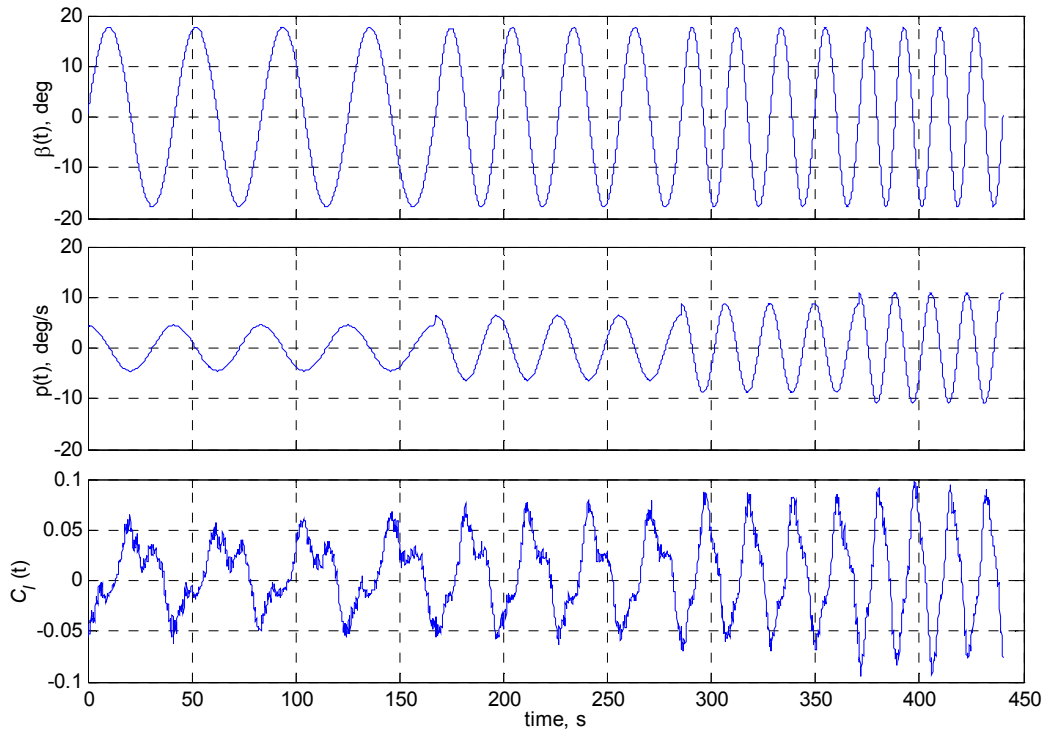

Figure 9b. Time histories of the large amplitude oscillatory data at $\alpha_{0}=$ $37.5^{\circ}, \beta_{0}=0^{\circ}$, and four frequencies, $\mathrm{k}=[0.066,0.095,0.131,0.160]$. 


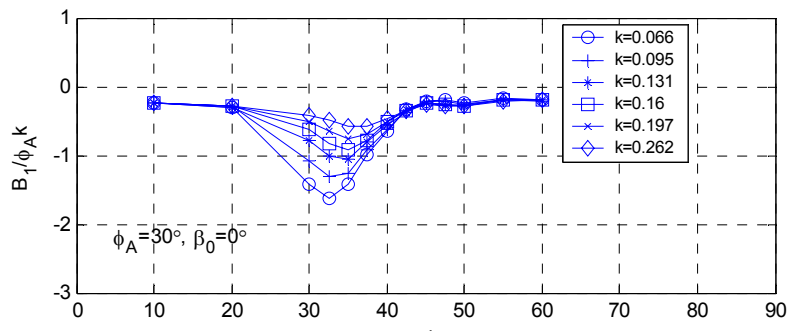

Figure 10. Variation of first-order harmonic coefficient with angle of attack and frequency.
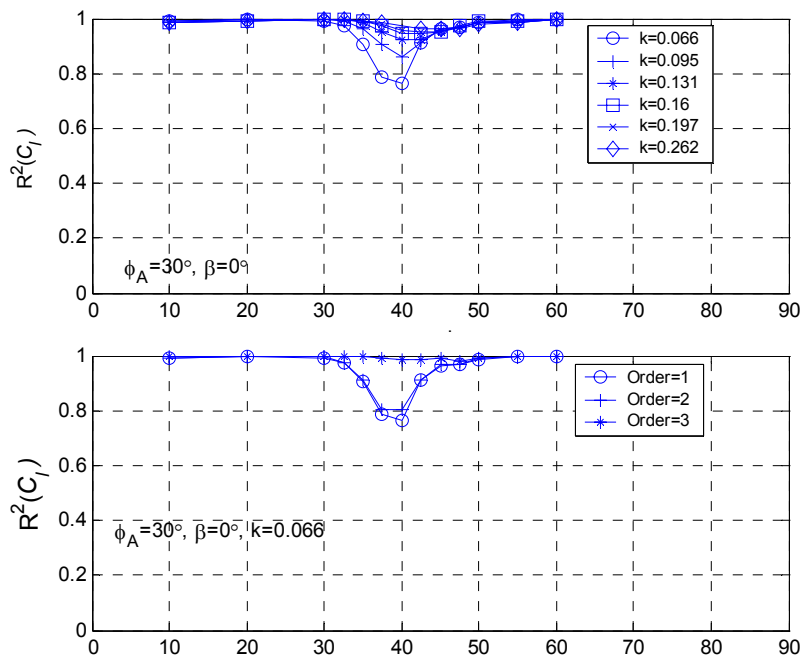

Figure 11. Variation of Multiple Corelation Coefficient with angle of attack, frequency, and order of harmonic coefficient. 

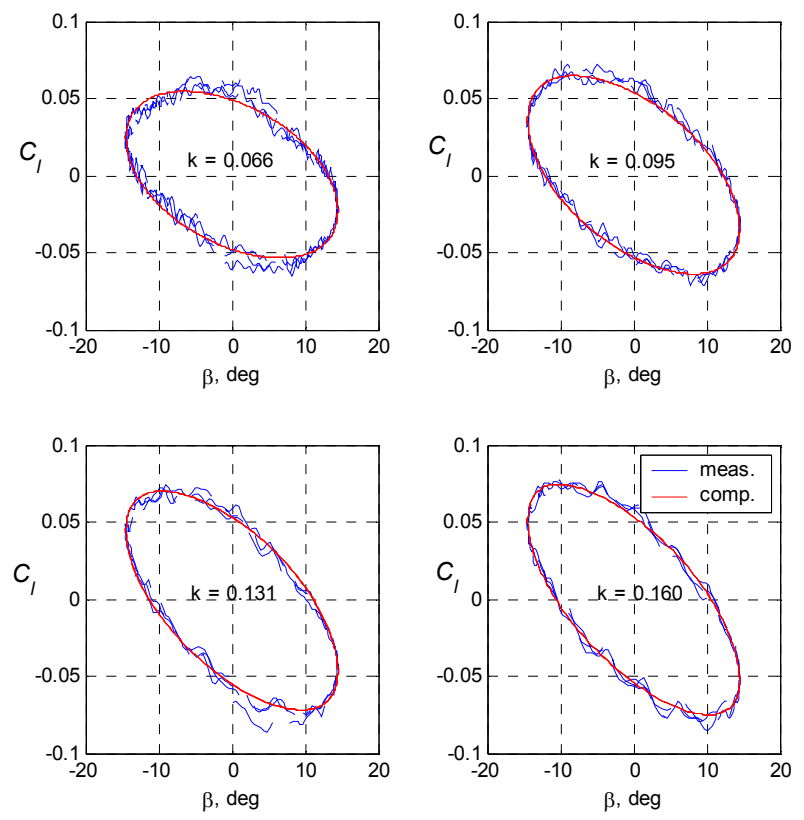

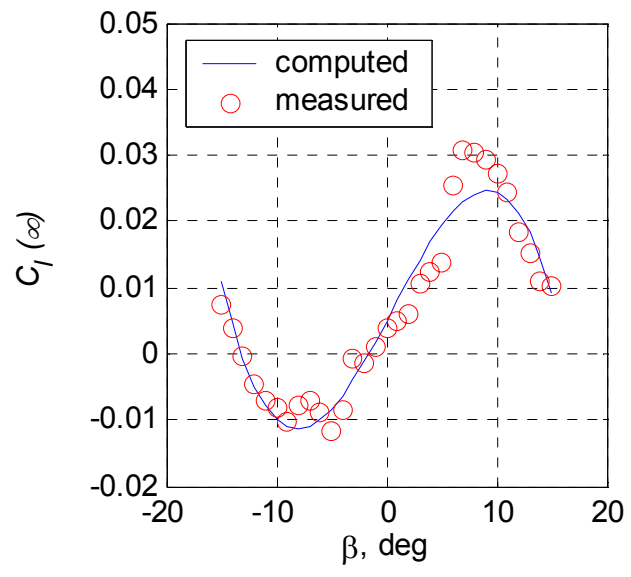

Figure 13. Comparison of measured and computed rolling-moment coefficient from $3^{\text {rd }}$ order polynomical at $\alpha_{0}=30^{\circ}$.

Figure 12. Comparison of measured and computed rollingmoment coefficient from relaxation method at $\alpha_{0}=30^{\circ}$.

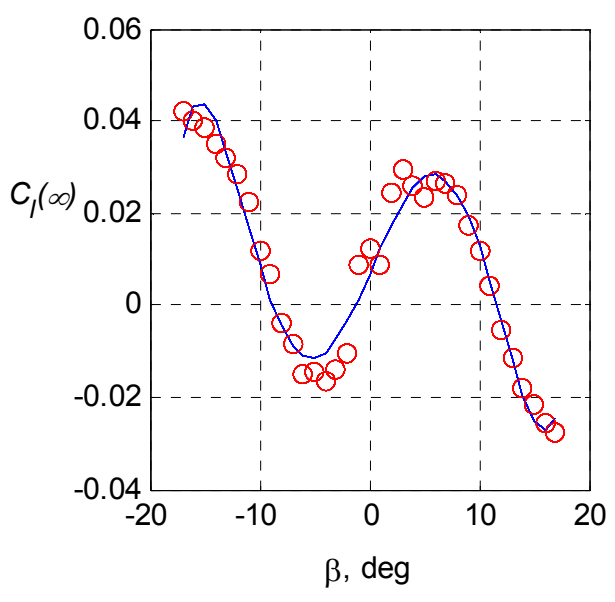

Figure 14. Comparison of measured and computed rolling-moment coefficient from $5^{\text {th }}$ order polynomial at $\alpha_{0}=37.5^{\circ}$.
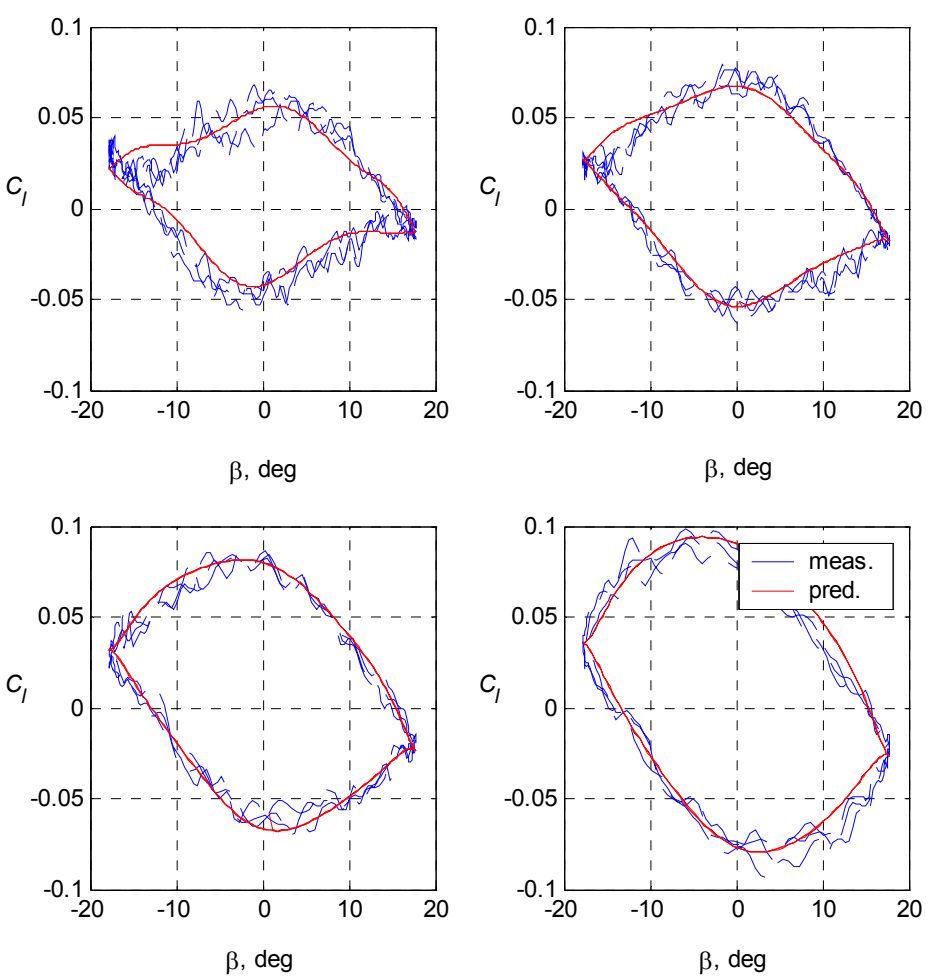

Figure 15. Comparison of measured and computed rollingmoment coefficient from relaxation method at $\alpha_{0}=37.5^{\circ}$. 